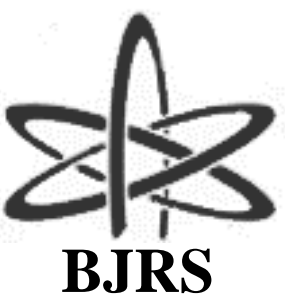

\author{
BRAZILIAN JOURNAL \\ $\mathrm{OF}$ \\ RADIATION SCIENCES \\ 09-02A (2021) 01-12
}

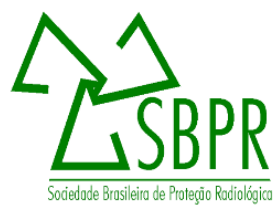

\title{
Study of gas pipelines cracks using transmission and Compton scattering
}

\author{
Marcela Ferreira Freitas, Cesar Marques Salgado \\ Instituto de Engenharia Nuclear \\ marcelafreita@gmail.com
}

\begin{abstract}
Most of the natural gas production is transported through pipelines that require periodic inspections to evaluate the structural integrity of the pipelines due to possible defects caused by degradation that can rupture causing leakage of the fluid causing major disasters. Based on this, the project presents a methodology for predicting cracks in pipe used in gas pipelines. The approximation is based on the principles of gamma densitometry to calculate the density of the pipe wall in order to investigate possible cracks. The natural gas fluid is found in such systems and interferes in the density calculations and therefore will be considered in the simulations. The detection system uses a narrow beam geometry appropriately, comprising gamma ray source $\left({ }^{137} \mathrm{Cs}\right)$ and $\mathrm{NaI}(\mathrm{Tl}) 3 \mathrm{x} 3$ " detectors for calculating transmitted and scattered photons. Different positioning angles of the detector are investigated. In this study, the MCNP-X code is used to perform the simulations, in order to develop a counting geometry. Simulations of different thicknesses of the crack were also used to determine the minimum thickness detected by the two $\mathrm{NaI}(\mathrm{Tl})$ detectors. Having equipment that can estimate cracks present in pipes used in gas pipelines, in addition to predicting their location can reduce costs and make a major contribution to this sector.
\end{abstract}

Keywords: gas pipelines, densitometry, gamma-ray gauge, Compton scattering, cracks.

ISSN: 2319-0612

Accepted: 2021-04-01 


\section{INTRODUCTION}

Due to the growing demand, natural gas is a source of energy that has been showing growth in the Brazilian energy matrix for use in industry, homes, motor vehicles and the electric sector. The development of this matrix is essential for industries to become competitive in order to increase operational efficiency and reduce costs. To do so, investments are needed in the expansion of the transmission network and in the distribution of this source of energy.

Much of the natural gas production is transported by means of gas pipelines that require periodic inspections to evaluate the structural integrity of the pipelines due to possible defects caused by degradation that may rupture causing leakage of the fluid causing large-scale environmental disasters with population damage and the economy of the local region. In Brazil, there are a total of 9,700 km of gas pipelines, 7,107 km operated by TRANSPETRO (TRANSPETRO, 2014) and $2,593 \mathrm{~km}$ operated by TBG (TBG, 2015), through 443 municipalities, with a transportation capacity of 130 million $\mathrm{m}^{3} / \mathrm{d}$ natural gas, making the control of gas pipeline integrity important to predict abnormality that could cause future risk to people and the environment.

Using conventional technology to perform preventive or corrective measures, crack detection can occur through internal pipeline monitoring by a variety of techniques, such as: instrumented, magnetically based Pipeline Inspection Gauge (PIG) is the most widespread tool for corrosion inspection of ducts. It uses changes in magnetic field to detect changes in duct thickness (Silva, 2015). However, the cost to the company for the launch and follow-up of the instrumented PIG passage in the pipelines is very high, in the order of US\$2,000.00 per km of pipeline (Carlos et al., 2002), besides the possibility of losing monitoring of the PIG when there is variation in the velocity of the fluid, above $3.0 \mathrm{~m} / \mathrm{s}$ and below $1.0 \mathrm{~m} / \mathrm{s}$ of that allowed for PIG monitoring.

In the context of Nuclear Techniques, a potential solution for the preventive control and evolution of cracks has been shown to be adequate to monitor and quantify the crack by means of the density of the material being analyzed, such as: gamma ray densitometry. The method is based on the change in attenuation of the gamma radiation caused by the presence of internal or external discontinuities when the radiation passes through the material and is recorded by specific detectors. Density measurement is based on the absorption of gamma radiation as it passes through the material to 
be analyzed. Absorption that is proportional to changes in material density indicates its density as the measurement path is kept constant.

The gamma-ray densitometry technique has been applied satisfactorily in many areas, such as: petrochemical, oil industry and mining. It has been used for flow measurements (Salgado et al., 2009); prediction of density (Achmad et al., 2004); thickness measurements (Berman et al., 1954) and inspection of pipeline surfaces (Anjos et al., 1989). This method of absorbing gamma rays can provide reliable measurements of the density of the analyzed material, improving accuracy. These advantages make these density meters very important in the industry and for this reason have been investigated and improved by many researchers.

However, in this type of measures difficulties are encountered, such as: extension and large diameter of the pipes, presence of fluids that due to differences in density interfere with the accuracy of density estimation. In contrast, in Compton scattering, the detector and the source can be arranged on the same side of the tube and an image of the cross-section of the duct can be directly obtained from the scattered radiation (Sharaf, 2001). Photons scattered alone from a well-defined volume of a sample contain information on the density of the material being analyzed (Arendtsz et al., 1995). Theoretical models were developed using the mathematical code MCNP-X based on simulations using the Monte Carlo method to develop a suitable counting geometry for crack detection by calculating density.

\section{GENERAL CONSIDERATIONS}

Devices based on nuclear technology allow detailed "observation" of flow characteristics, especially density, distribution of the solid particles, concentration in the medium and the speed of displacement, among others. The method is based on the attenuation of a gamma-ray monoenergetically transmitted/scattered beam by a tubing containing fluid being the signal recorded by detector(s). The two methods of measurement: transmitted and scattered beams will be tested.

In the transmitted beam, a sealed, shielded monoenergetic gamma radiation source with sufficient energy to penetrate and be transmitted throughout the tube is positioned on one side of the pipeline and a $\mathrm{NaI}(\mathrm{Tl})$ scintillation detector is generally installed, diametrically opposite the source for the 
purpose of recording the counting rate. The recording of the signal in the detector is proportional to the transmission of the gamma rays due to the thickness of the duct, the fissure and the flow existing inside the duct. The duct can be represented by an absorber system composed of "n" layers of materials with different thicknesses and attenuation coefficients. Considering a simplified model with the gas phase moving in a duct with cylindrical straight section, knowing the dimensions and characteristics of the duct and of the transported fluid, the intensity of the transmitted beam can be calculated by the law of Beer-Lambert's with Equation 1:

$$
\mathrm{I}(\mathrm{E})=\mathrm{I}_{\mathrm{o}}(\mathrm{E}) \cdot \exp -\left(\mu_{\text {duct }} x_{\mathrm{D}}+\mu_{\text {gas }} x_{\mathrm{G}}+\mu_{\text {fissure }} x_{\mathrm{F}}\right)
$$

Where I is the transmitted intensity of gamma rays (photons $\mathrm{cm}^{-2} \cdot \mathrm{s}^{-1}$ ), Io is the initial incident intensity of gamma rays from the source (photons. $\mathrm{cm}^{-2} \cdot \mathrm{s}^{-1}$ ), $\mathrm{E}$ is the incident radiation energy, $\mathrm{X}$ is the material thickness D duct, $G$ gas, $F$ fissure $(\mathrm{cm})$, and $\mu$ is the total attenuation coefficient D duct, $G$ gas and $\mathrm{F}$ fissure, $\left(\mathrm{cm}^{-1}\right)$.

In Compton scattering, where the energy of the scattered photon is smaller than that of the incident photon; that is, the incident photon transfers a fraction of its energy to an electron from the weakly bound layers of an atom resulting in a photon of reduced energy, E $\gamma$ '. From the conservation of momentum and energy equations, we calculate the energy of the scattered photon (E $\left.\gamma^{\prime}\right)$ which has different direction of the incident photon and is calculated by Equation 2.

$$
E \gamma^{\prime}=\frac{h v}{1+\frac{h v}{m_{o} c^{2}}(1-\cos \theta)}
$$

Where $\mathrm{h} v$ is the incident photon energy $(\mathrm{keV}), \theta$ is the scattering angle relative to the initial direction, $\mathrm{m}_{\mathrm{o}}$ is the resting mass of the electron $(\mathrm{kg}), \mathrm{c}$ is the speed of light $\left(\mathrm{m} . \mathrm{s}^{-1}\right)$ and $\mathrm{m}_{\mathrm{o}} \mathrm{c}^{2}$ is the resting energy of the electron $(511 \mathrm{keV})$.

When the energy of the incident photon is much greater than the energy of binding of the orbital electrons, Compton scattering overrides the other two interactions (photoelectric and Rayleigh). In the case of photons with energies $(100 \mathrm{keV}-10 \mathrm{MeV})$ materials with low atomic number $(\mathrm{Z} \leq 40)$, 
Compton scattering is the largest mechanism of interaction. The probability of Compton scattering is directly proportional to the photon energy and inversely to the atomic number. The number of scattered photons reaching the detector can be obtained by Equation 3.

$$
S=\Phi_{o}(E) \varepsilon(E) t \exp \left(-\int_{l_{1}} \mu\left(E_{\gamma}\right) d l\right) \frac{d_{e} \sigma^{K N}}{d \Omega} \frac{\rho N_{A} Z}{A} \exp \left(-\int_{l_{2}} \mu\left(E_{\gamma^{\prime}}\right) d l\right) d V d \Omega
$$

Where $t$ is the count time $(s), I_{0}(E)$ is the incident photon flux with energy $(E), d_{e} \sigma^{K N} / d \Omega$ is the Klein-Nishina differential shock section in energy (E) for a free electron, which is the scattering probability of a photon, $d \Omega$ is the solid angle subtended by the detector as seen from the interaction point, $\rho$ is the density, $\mathrm{Z}$ is the atomic number, $\mathrm{N}_{\mathrm{A}}$ is the Avogadro's number, $\mathrm{A}$ is the mass number of the material on analysis, $\mu_{1}$ e $\mu_{2}$ are the linear attenuation coefficients for primary and scattered photons within the sample, $\varepsilon$ is the detector photopeak counting efficiency in scattered photon energy, $l_{1}$ e $l_{2}$ are the path lengths of the photons in the source sample to the scattering center and back to the detector and $\mathrm{dV}$ is the differential volume considered for radiation and its interaction with matter.

In the case of a homogeneous sample and geometry with point source, the terms of the integrals are constant. In addition, the Klein-Nishina differential shock section will be constant for a fixed geometry, a given incident photon energy and flux. Therefore, the counting rate depends only on the density of the material examined and information obtained by this technique is strongly dependent on this density, so that the variation of density within the sample can be monitored.

\section{MATERIALS AND METHODS}

\subsection{Simulated Geometry}


The simulation consisted of a carbon steel pipeline $5 "$ in diameter and $0.17 "$ thick, inside the pipeline the natural gas fluid was used, as reference was the natural gas delivered to Companhia de Gas de São Paulo (COMGAS), with a density of $0.766 \mathrm{~kg} \cdot \mathrm{m}^{-3}$ (@ $20^{\circ} \mathrm{C}, 1 \mathrm{~atm}$ ). A source of ${ }^{137} \mathrm{Cs}$ $(662 \mathrm{keV})$ with narrow beam was appropriately used in the simulation with the angle of $4.29^{\circ}$ divergence. Three lead collimated 3"x 3" Nal (Tl) detectors were properly positioned, the first at $180^{\circ}$ (D1), the second at an angle of $30^{\circ}$ (D2) and the third at $60^{\circ}$ (D3) as shown in Figure 1.

Figure 1: Simulated Geometry

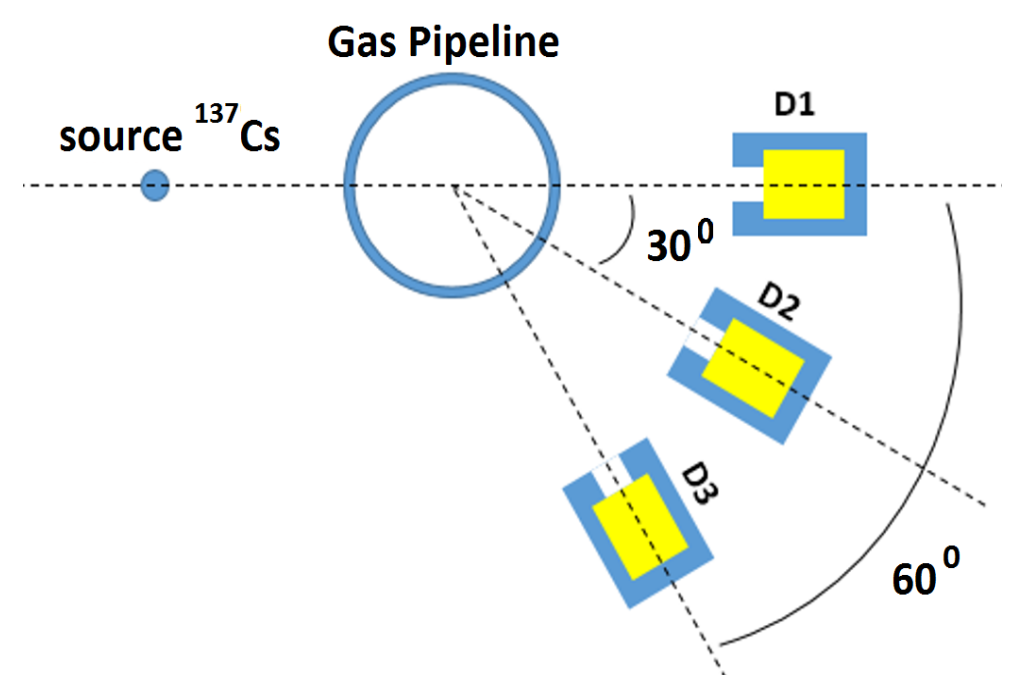

The detector D1 was used to measure the attenuation of the transmitted beams, and the others were used to measure the scattered beams.

\subsection{Potentiality of the code in scattering calculation}

In order to evaluate the potential of the MCNP-X code in the scattering calculation, a geometry formed by a solid aluminum tube was developed, simulating a scattering bar with a diameter of 1 $\mathrm{cm}$. The same ${ }^{137} \mathrm{Cs}$ source $(662 \mathrm{keV})$ was simulated appropriately with the angle of $4.29^{\circ}$ divergence and a scatter detector was positioned at $30^{\circ}$ and $60^{\circ}$. The results obtained were compared with theoretical data obtained by Equation 2. 
The study also considered the influence of the scattering of the 5"carbon steel pipeline and the contribution of the natural gas fluid inside this pipeline, in the D2 and D3 detectors.

\subsection{Determination of cracks}

For the study of the fissures in the pipelines, fissures were added in the rectangular (parallelogram) format of $3 \mathrm{~mm}$ of thickness, $50 \mathrm{~mm}$ of height, the width was changed from $2 \mathrm{~mm}$ to $16 \mathrm{~mm}$, in steps of $2 \mathrm{~mm}$. The first simulation was done without the crack. As shown in Figure 3.

Figure 3: Crack dimensions and location of the crack in the simulated geometry

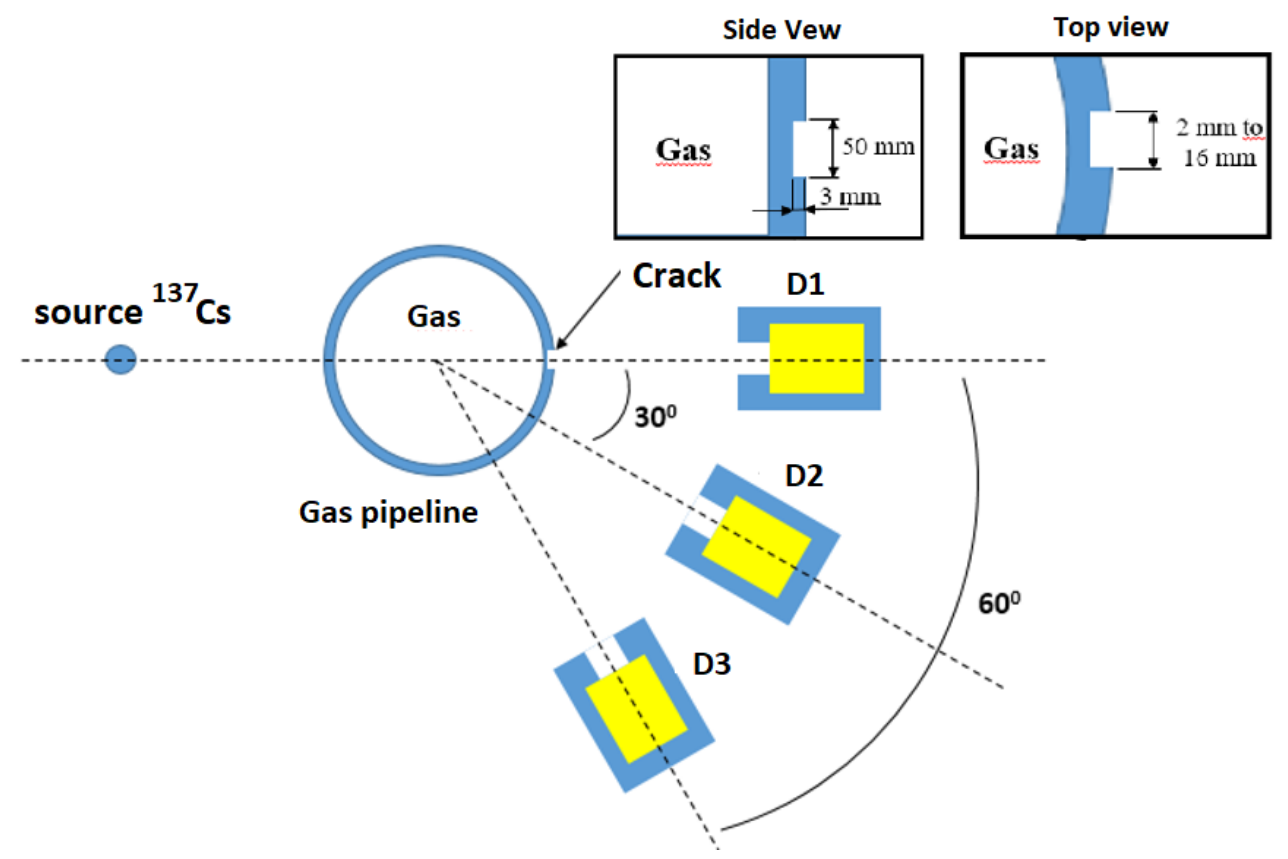

\section{RESULTS AND DISCUSSION}

In the analysis of the study of the pontenciality of the code in the scattering calculation, it can be observed that according to Equation 2, the scattered energy referred to $662 \mathrm{keV}$ at $30^{\circ}$ and at $60^{\circ}$ is $564 \mathrm{keV}$ and $411 \mathrm{keV}$, respectively. In the simulation performed, the scattering energy detected by 
the respective detectors D2 and D3 were $570 \mathrm{keV}$ and $410 \mathrm{keV}$, validating the generated geometry for the simulation study in the MCNP-X code, the results are show in Figure 4.

Figure 4: Scattering photons detected by detectors D2 (30 ) and D3 (60 )

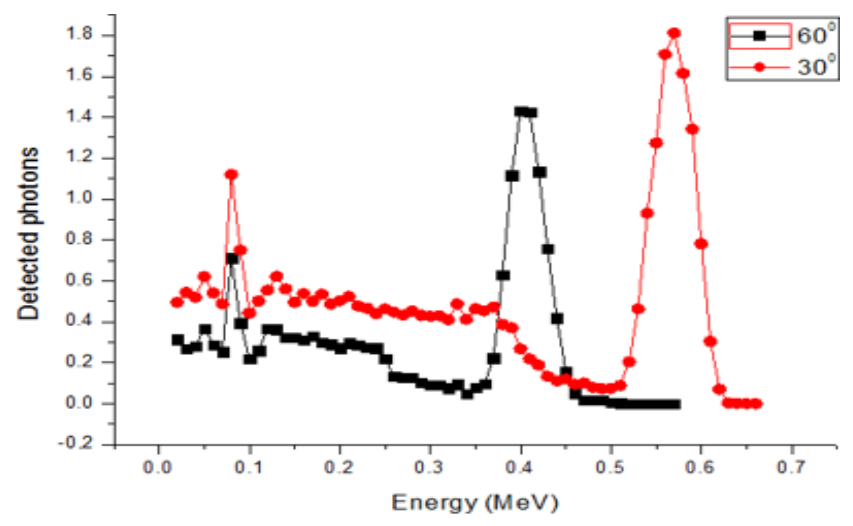

In Figure 5 show the influence of the carbon steel duct on the scattering. As can be observed, the scattering generated by the carbon steel is distributed in two peaks, $480 \mathrm{keV}$ and $610 \mathrm{keV}$ for the detector D2 and $310 \mathrm{keV}$ and $480 \mathrm{keV}$ for the detector D3.

Figure 5: Scattering photons detected by the detector D2 (a) and by the detector D3 (b)

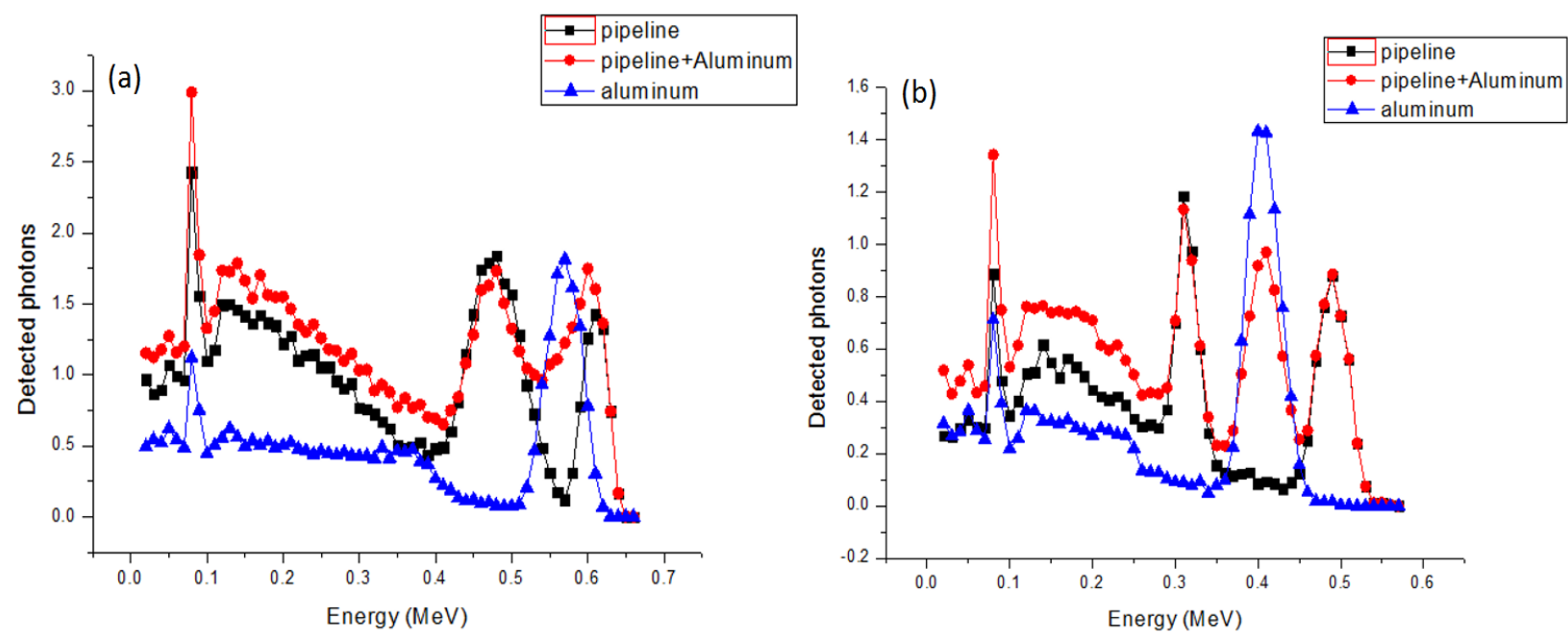


Due to the low density of the natural gas fluid of $0.766 \mathrm{~kg} / \mathrm{m}^{3}$ in relation to the dry air, no contribution was observed in the scattering, according to Figure 6.

Figure 6: Contribution of the natural gas fluid to the scattering photons in the detector D2 (a) and in the detector D3 (b)
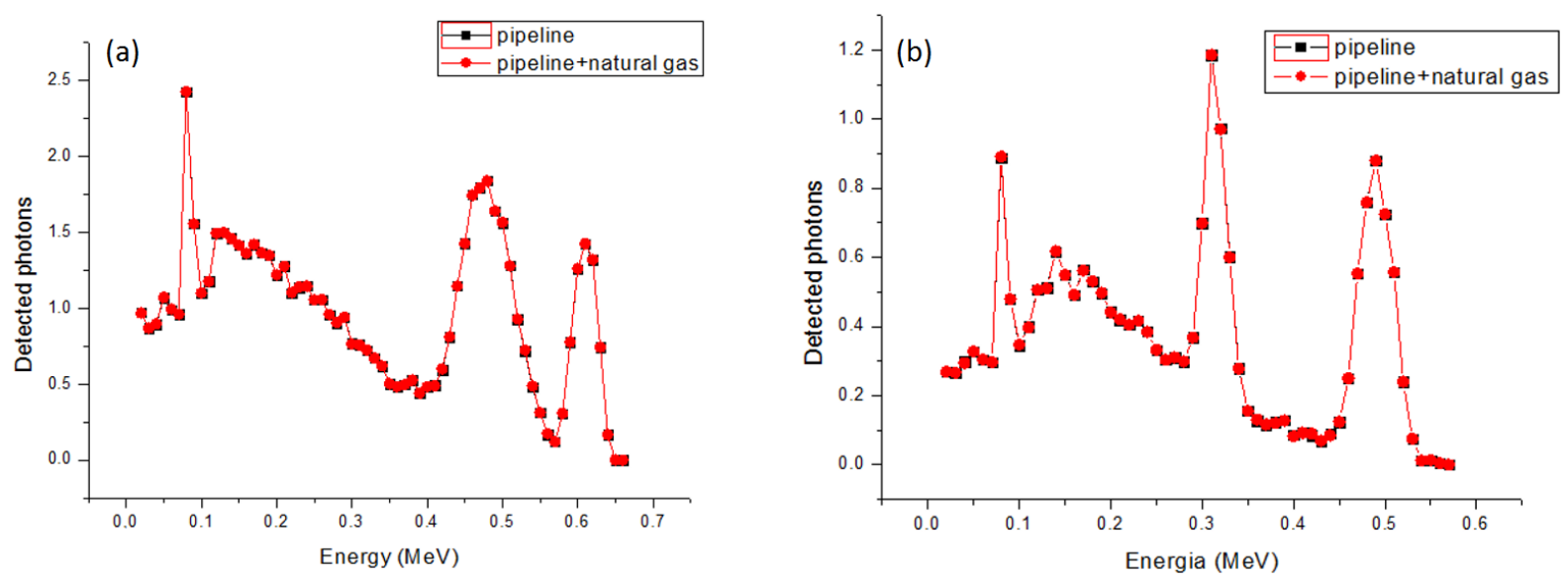

\subsection{Results in the analysis of cracks:}

In the detector D1 it was possible to observe the decrease of the attenuation of the transmitted beams, due to the increase of the crack in the energy of $662 \mathrm{keV}$, according to Figure 7, that is, the larger the width of the crack, the greater the number of beams transmitted and detected by D1.

Figure 7: Photons detected by detector D1 as a function of crack width 


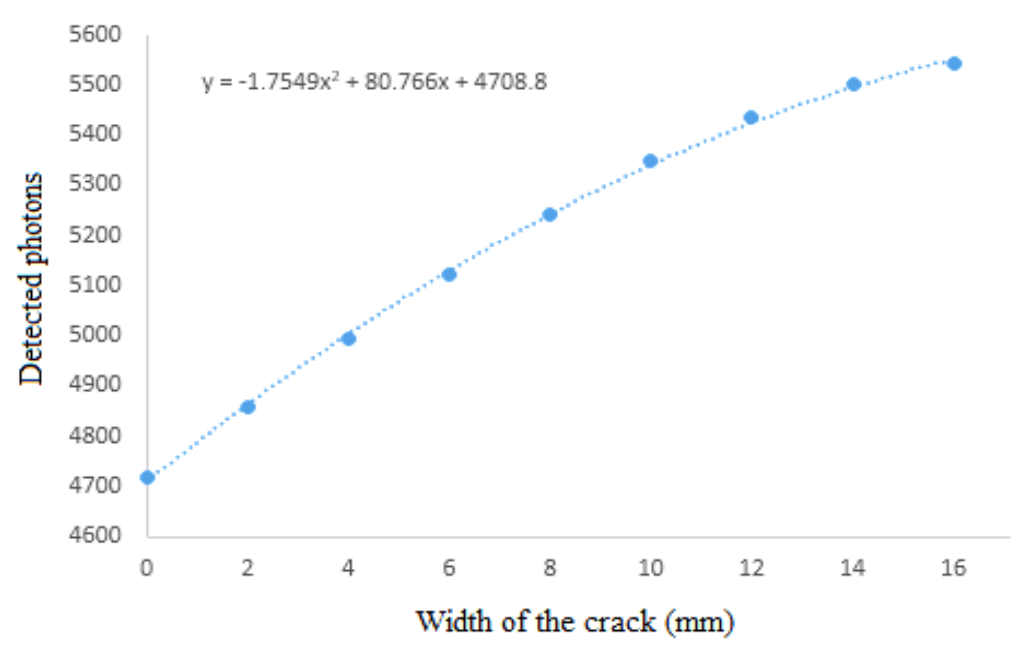

As can be observed, it was possible to define a relation between the crack width as a function of the number of photons detected by detector D1, defined by Equation 4 .

$$
y=-1.7549 x 2+80.766 x+4708.8
$$

Where $\mathrm{y}$ is the numbers of photons detected by detector D1 and $\mathrm{x}$ is the crack width.

In the detectors D2 and D3, the "behavior" of the scattering photons in relation to the crack width variation was analyzed, and as can be observed in Figure 8, there was an increase in the number of photons scattered as a function of reduction of the crack width, as can best be seen at the energies of $480 \mathrm{keV}$ for the D2 detector and $310 \mathrm{keV}$ for the D3 detector.

Figure 8: Scattering photons detected by detector D2 (a) and by detector D3 (b) 

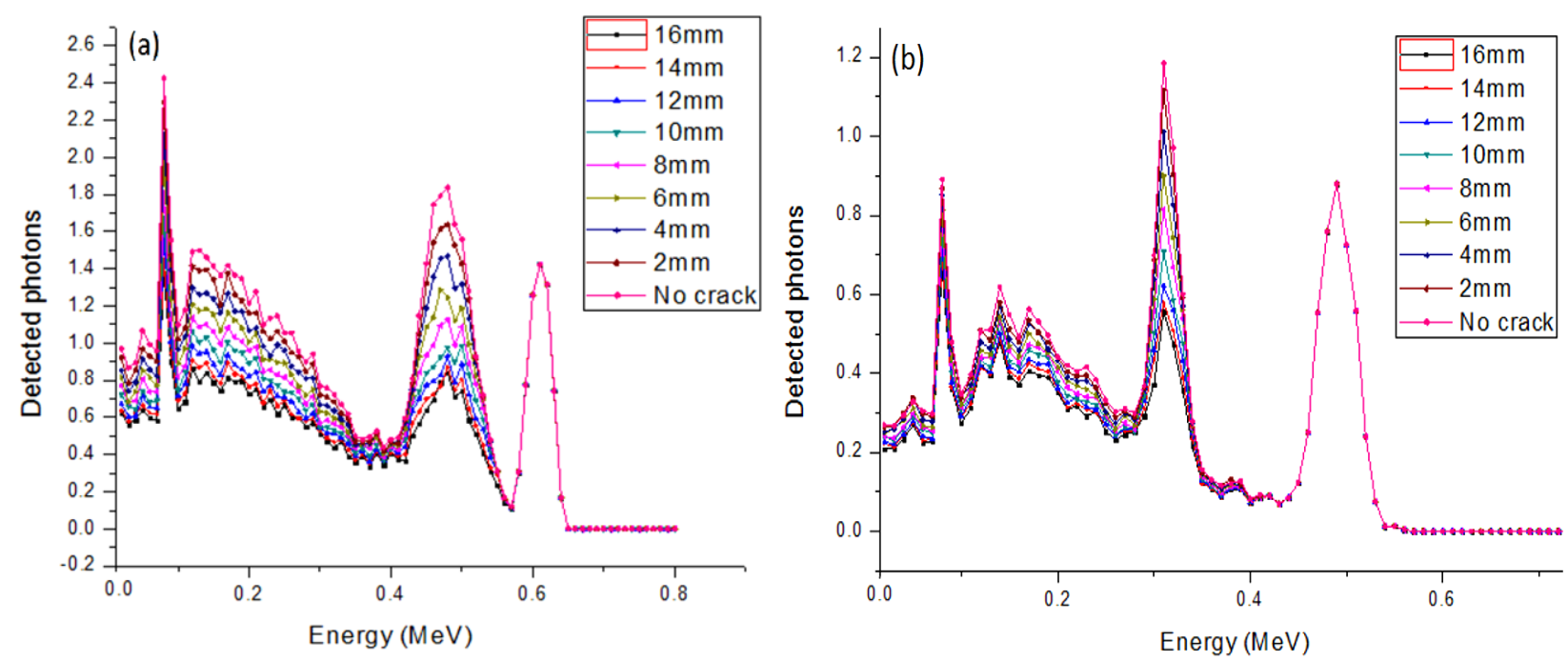

The scattered photons were detected by the D2 and D3 detectors, at the energies of $480 \mathrm{keV}$ and $310 \mathrm{keV}$ respectively, in function of the crack width, according to Figure 9.

Figure 9: Scattering photons detected by detector in function of the crack width, at the energies: (a) $480 \mathrm{keV}$ for D2 and (b) $310 \mathrm{keV}$ for D3
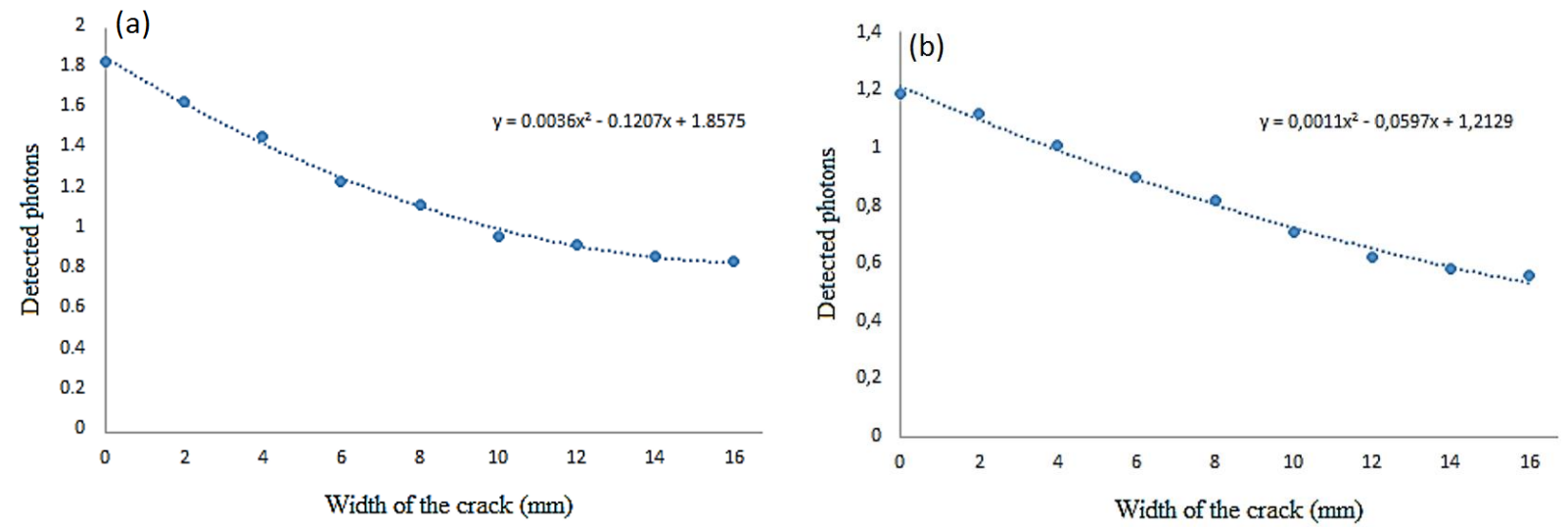

It was possible to define a relation between the crack width as a function of the number of scattering photons detected by detectors D2 and D3, defined by Equation 5 and 6 respectively. 


$$
\begin{aligned}
& y=0.0036 x^{2}-0.1207 x+1.8575 \\
& y=0.0011 x^{2}-0.0597 x+1.2129
\end{aligned}
$$

Where $\mathrm{y}$ is the numbers of scattering photons detected by detector and $\mathrm{x}$ is the crack width.

\section{CONCLUSION}

The MCNP-X code was shown to be an important and useful tool to continue the studies of cracks analysis, given that it was possible to carry out the surveys of the scattering energies correctly. The model developed in the code represented satisfactorily the scattering beam.

It was possible to identify that the nuclear techniques are sensitive for determination of crack size, which in this study was $2 \mathrm{~mm}$ to $16 \mathrm{~mm}$, being possible the differentiation between them, by means of the adjusted curves. For future work, will be changed the position of the crack and will be used Artificial Neuronal Networks (RNAs) to identify its location.

\section{ACKNOWLEDGMENT}

I hereby express my gratitude to the Institute of Nuclear Engineering, without which this work would not have been possible.

\section{REFERENCES}

[1] ACHMAD B.; HUSSEIN E. M. A. An X-ray Compton scatter method for density measurement at a point within an object, Applied Radiation and Isotopes, 2004. p. 805-814.

[2] ANJOS M. J.; LOPES R. T.; BORGES J. C. Compton scattering of gamma-rays as surface inspection technique. Nuclear Instruments and Methods in Physics Research Section, 1989. p. 535-538. 
[3] ARENDTSZ N. V.; HUSSEIN E. M. A. Energy-spectral Compton scatter imaging. I. Theory and mathematics. IEEE Transactions on Nuclear Science NS-42, 1995. p. 2155-2165.

[4] BERMAN A. I.; HARRIS J. N. Precision Measurement of Uniformity of Materials by Gamma Ray Transmission, Review Scientific Instruments, 25, 1954. p. 21-29.

[5] OLIVEIRA C. H. F., CAMERINI C. S. Instrumented PIG of PETROBRAS results and prospects, XXI National Congress of Non-Destructive Testing, 2002 p.9.

[6] SALGADO C. M.; BRANDÃO L. E. B.; NASCIMENTO C. M. N. A.; SCHIRRU R.; RAMOS R.; SILVA A. X. Prediction of volume fractions in three-phase flows using nuclear technique and artificial neural network, Applied Radiation and Isotopes, 67, 2009. p. 1812-1818.

[7] SHARAF J. M. Practical aspects of Compton scatter densitometry. Applied Radiation and Isotopes 54, 2001. p. 801-809.

[8] SILVA M. A. The use of PIGs in the inspection and maintenance of pipelines. Technology \& Information Magazine - ISSN 2318-9622, 2015. p.38-52.

[9] TRANSETRO, Natural Gas. 2014. Available at: <http://www.transpetro.com.br/pt_br/areasde-negocios/gas-natural.html>. Last accessed: 27 may. 2017. 